Sandaruwan, I.P.T., Chandanie H. and Janardana, J.A.B., 2021. Barriers in practicing life cycle costing techniques experienced by Sri Lankan quantity surveyors. In: Sandanayake, Y.G., Gunatilake, S. and Waidyasekara, K.G.A.S. (eds). Proceedings of the $9^{\text {th }}$ World Construction Symposium, 9-10 July 2021, Sri Lanka. [Online]. pp. 160-170. DOI: https://doi.org/10.31705/WCS.2021.14. Available from: https://ciobwcs.com/papers/

\title{
BARRIERS IN PRACTICING LIFE CYCLE COSTING TECHNIQUES EXPERIENCED BY SRI LANKAN QUANTITY SURVEYORS
}

\author{
I.P. Tharindu Sandaruwan ${ }^{1}$, H. Chandanie ${ }^{2}$ and J.A.B. Janardana ${ }^{3}$
}

\begin{abstract}
Life Cycle Costing (LCC) is a tool, which can evaluate all the building-related costs over a specified period of time, as mentioned in the agreed scope. Though there are number of benefits, LCC practices of consultants in the building industry are claimed to be weak. Hence, the aim of this research is to investigate barriers in practicing LCC techniques experienced by Sri Lankan quantity surveyors. Accordingly, a mixed approached was followed in data collection through a questionnaires survey and a series of expert interviews. However, in Sri Lanka, building construction consultants are not practicing the LCC concept to a greater extent. This research identified, lack of awareness on the LCC tool by employers and practitioners, lack of knowledge on LCC and lack of previous data as the major barriers for practicing LCC techniques in local context. It was suggested that enhancing the practice of LCC through conducting various awareness programme on LCC concept, build up and maintain the proper database and introducing user friendly tools, applications, calculations methods, guidelines and regulations, will lead to avoid above mentioned barriers, which will ultimately enhance the proper practice of LCC concept in the Sri Lankan quantity surveyors.
\end{abstract}

Keywords: Building; Construction; Life Cycle Costing (LCC); Sri Lanka.

\section{INTRODUCTION}

Investing significant sum of money in the construction sector requires a careful appraisal to ensure that optimum use is being made of the sums invested. Yet often, investors or owners focus only on the initial cost when they make decisions and then they tend to ignore future maintenance costs and operation costs (Davies, 2004). LCC is a tool which also assists to determine possible cost reductions throughout the project life cycle (Bull, 1993). LCC is an approach to prior stage building project evaluation, that seeks to determine total expenditure of a facility by analysing all initial costs of purchasing and other associated costs including operational and maintenance costs throughout the design life of proposed building project (Kirk and Dell'Isola, 2003). According to Cole and Sterner (2000), discounting of future costs to present values is one of the key aspects in performing LCC. The authors state that this aspect of LCC enable the design option

\footnotetext{
${ }^{1}$ School of Civil Engineering and Built Environment, Liverpool John Moores University, UK, sandaruwantharindu12@gmail.com

${ }^{2}$ Department of Building Economics, University of Moratuwa, Sri Lanka, chandanieh@uom.lk

${ }^{3}$ School of Civil Engineering and Built Environment, Liverpool John Moores University, UK, bihara.j@sliit.lk
} 
comparison to be made on a level playing field (Cole and Sterner, 2000). In addition, LCC can be used to predict the cash flow of an asset, for budgeting, for cost planning, for tendering, and for cost reconciliation. Further, LCC is a useful tool in design option appraisal and in assessing the present and likely maintenance costs in the future (Kelly and Hunter, 2009). Furthermore, ultimate conclusions from LCC output represents the total cost commitment of a facility, evaluation of various alternatives leading to reduce the unnecessary costs, enhance the cost transparency, recognise the different cost drivers and identification of risk factors as benefits of using the LCC techniques (Knauer and Moslang, 2005).

In the construction sector LCC is mostly used by the consultants as a tool for various purposes. Yet, in Sri Lanka, construction consultants are claimed to be heavily backward in practicing these LCC techniques. Therefore, this study intends to investigate barriers in practicing life cycle costing techniques experienced by Sri Lankan quantity surveyors.

\section{LITERATURE REVIEW}

\subsection{LIFE CYCLE COSTING (LCC)}

LCC has been defined as a technique which can use to measure all costs related to construction, operation, and maintenance of a construction project over a particular timeframe (Heralova, 2017).

Accordingly, the LCC technique gives an emphasis to a whole or the total cost approach consumed during the acquisition of a capital cost project or asset, rather than merely concentrating on the initial capital costs alone. Hence, LCC facilitates considering optional solutions for different variables involved and sets up hypotheses to test the confidence of the results achieved. Taking into account the initial capital costs, maintenance costs and replacement or the salvage costs and expressing these costs in comparable terms, this LCC as an asset management technique allows the operating costs of premises to be evaluated at frequent intervals, in which also can be recognised as its unique advantages (Ashworth et al., 2013). According to Ellram (1995), LCC is more concerned about capital or fixed assets (Ellram, 1995). Differently, another study stated that LCC can be used for any sort of a product (Aseidu and Gu, 1998). However, the main motivation for the implementation of LCC methods in the construction industry is LCC being identified as a tool for decision making (D'Incognito et al., 2015). Many professionals use LCC techniques for different applications leading to different purposes. Decision making at the design stage, to determine the performance impact throughout the lifespan of buildings, selection of most cost-effective project from a series of alternatives and assessment of new installation services against existing installation services can be recognised as some generic purposes of application of LCC techniques in the construction industry (Dale, 1993).

Furthermore, ultimate conclusions from LCC assessment output represents the total cost commitment of a facility which can be effectively utilise for reduction of building ownership cost, evaluating economic aspects of a project, enhancing the risk management process, monitoring the cost performance of a project, control design development, identify the cost of project, enhance the cost transparency, and also in recognising the different cost drivers as key benefits of application of LCC techniques (Knauer et al., 2005). 


\subsection{BARRIERS FOR PRACTICING LCC}

Akhlaghi (1987) conducted a case study, which was carried out to compare the LCC in five factory buildings in typical companies. In the study the authors analysed and measured each building's LCC and each case showed optimal performance of costs-inuse. During the study, the authors found a major barrier on the application of LCC as not having sufficient appropriate database, to cover the cost and performance (Akhlaghi, 1987).

In UK, another research study was conducted by Hunter et al., (2005), which carried out to develop a framework document and WLC input technique for use in public sector to enable QS. The methodology of this study comprised of four (04) phases. First stage was to have investigative interviews with the industrial expertise. The second stage is the comprehensive literature review. The presentation of WLC IT Tool is the third stage. The fourth stage was the demonstration at the annual SCQS conference for feedback. In this study the researchers faced various challenges during the study. Conclusively, the authors determined that major challenges are in collecting the LCC (capital, facilities management, and disposal) data. The lack of previous data of the building elements and services is the reason behind this challenge (Hunter et al., 2005). Samani et al. (2018) conducted a research aimed at comparing the LCC analysis of prefabricated composite buildings and masonry buildings in USA. The authors considered the four (04) life cycle stages; construction operation, maintenance and demolition, and buildings in the cities of Los Angeles, El Paso and San Francisco. The results of their study provided that, the significance of construction cost for both prefabricated and masonry structures are and higher value of maintenance and demolition costs of the prefabricated buildings. In addition, they identified that, the inadequate previous data and necessary hierarchy level of stakeholders as barriers in implementing LCC (Samani et al., 2018).

Higham et al. (2015) conducted a research aimed at evaluating LCC use in the UK practice. The authors used both qualitative and quantitative approaches for their study. Qualitative approach was used to understand the context-specifics whereas quantitative approach was used to test hypothetical generalisations. Meanwhile, this study showed, lack of awareness on the LCC tools by the employers and practitioners, unreliability of data, need of employers to keep the budget within short-term horizons and lack of common methods as inhibitors of the implementing LCC as an early phase project evaluation tool in the UK (Higham et al., 2015). In another study conducted by Herlova (2017), which carried out to study significant influence of LCC to the feasibility study in the government sector construction projects. This study identified and summarised the opportunities and challenges of using LCC in an early phase by means of a literature review and case studies. Accordingly, lack of industrial standards for reporting LCC and lack of previous cost data were identified as barriers to LCC implementation (Heralova, 2017).

D'Incognito et al. (2015) conducted a study to identify the actors and barriers to the adoption of LCC and LCA techniques in the construction industry. The study comprised two stages. In the first stage, they reviewed the previous research and the evaluation was done using content analysis. In the second stage, they designed a questionnaire survey based on the content analysis results. The questionnaire survey was conducted with selected professionals and industry experts in LCC and Life Cycle Analysis in construction sector. This study found that the organisational culture as the most 
significant barrier, when adopting LCC and LCA in the built environment. In addition, software tools, regulations and standards, data and information, approach and methodology were identified as the technical barriers for the same. Furthermore, this study revealed, incentive taxes and high costs of implementation as the financial barriers (D'Incognito et al., 2015). Korpi and Ala-Risku (2008) reviewed reports on LCC applications to provide an overview of LCC uses and implementing feasibility in the built environment. In the study the authors found that the characteristics of the operating environment encourages the implementing of LCC, purposes for calculating LCC, how do LCC implementations conform to the different methods, and how do the characteristics of the operating environment affect to the methods used in LCC analysis. In addition, the authors mentioned that lack of reliable data, lack of formal guidelines and lack of standards are the reasons for the slow adoption of the LCC (Korpi and Ala-Risku, 2008).

Lindholm and Suomala (2007) conducted a research aimed to discuss LCC management and practical challenges related to collecting adequate data and practicing long-term cost management in an uncertain environment. In this study the authors determined; inconsistent data collection, scarce of the LCC practice by employers and practitioners, different opinions of stakeholders as the major challenges in adopting LCC in built environment (Lindholm and Suomala, 2007). A generic framework for collecting whole life cost data for the building industry was presented by El-Haram et al. (2002). To achieve the aim, the study was conducted in six (06) levels as project level, phase level, category level, element level, and task level. In each level the authors found the data used to calculate WLC. The study determined that building consistent data on the execution of building elements and services, difficulties in arriving conclusions on LCC process of a building and, inadequate data of the building elements and services as major problems in LCC adoption in construction industry (El-Haram et al., 2002).

Dale (1993) determined in his study that, inadequate joined up thinking regarding the life cycle construction process of the building or the project, and fragmented nature of the built environment sector are the major problems to practice the LCC in construction industry (Dale, 1993). In addition, Cole and Sterner (2000) identified organisation structure as the main restricted reason for LCC adoption in public sector (Cole and Sterner, 2000). Furthermore, Steen (2005) conducted a research to investigate the possibilities of using LCA results to identify and estimate environmental costs or benefits in an LCC. In this study, the author mentioned that lack of enforcement capacity, lack of knowledge of who caused what damages to whom, lack of regional and global consensus as the preventing reasons when practicing this principle (Steen, 2005).

According to Norman (1990), the most difficult barrier to the implementation of LCC in the built environment is the unavailability of a useable and reliable data base. Positively, the study mentioned of many professionals and government bodies who are now involved in building data bases to in overcoming this barrier (Norman, 1990). Olubodun et al. (2010) conducted a research aiming to appraise the levels of application of LCC in the UK construction industry. The study revealed, lack of understanding on LCC, lack of standardised methodology, complex process, deficiency of accuracy in results and, artificiality of the process as barriers for implementation of LCC in the UK construction industry (Olubodun et al., 2010). According to Bruce-Hyrkas et al. (2018), difficulties in understanding, information is not timely for the design process, non-availability of building LCC background data, enabling non LCC experts to do the calculations reliably, 
inability to use existing building data efficiently and heterogeneous requirements of various certifications are the challenges of performing LCC in building construction industry (Bruce-Hyrkas et al., 2018).

In 2017, Schmidt and Crawford carried out a research to develop an integrated framework for assessing the life cycle of greenhouse gas emissions and LCC of buildings. Meanwhile, the research identified lack of transparent input data, lack of transparent calculations, and lack of early-stage design applications and lack of adjustable personal parameters as the major challenges to implementation of LCC approach (Schmidt and Crawford, 2017). According to Opoku's (2013) work, there are several barriers in practising LCC in the construction industry; i.e. not requested by employers, lack of consistent and reliable data on cost and performance, lack of interest/motivation from the employers, lack of understanding on LCC, lack of standard methods for practising LCC, and inadequate data on durability (Opoku, 2013). In Malaysia, another study conducted was by Khiyon and Mohamed (2018) to investigate the barriers and drivers of whole life cycle costing of sustainable facility management for PPP/PFI projects in Malaysia. The study revealed lack of incentives, lack of standards and methods, lack of motivation and inconsistency in underlying methodology and philosophy as the main barriers of implementation LCC in sustainable facility management for PPP/PFI projects in Malaysia (Khiyon and Mohamed, 2018).

The literature discussed above lead to identify the barriers for the built environment in practicing LCC techniques. The important barriers which affect the practicing of LCC in the construction sector are summarised in Table 1.

Table 1: Barriers affecting practising LCC in built environment

\begin{tabular}{cc}
\hline Barriers & References \\
\hline Lack of previous and unrealistic data & {$[1],[2],[3],[4],[5],[6],[7]$,} \\
& {$[8],[9],[12],[15],[16],[17]$}
\end{tabular}

Lack of software tools

[6]

Lack of awareness on the LCC tool by employers and

[4], [13], [16] practitioners

Lack of common methods in calculating LCC

Lack of industrial standards for reporting the LCC

Lack of knowledge on LCC

Lack of formal guidelines

Lack of transparency in calculations

Lack of motivation / lack of interest from the employers

Lack of regulations and standards

[16], [17], [18]

[6], [17]

Need of employers to budget within short-term horizons

Different organizational cultures

Complexity in approach and methodology

[6], [13], [17]

Incentive taxes

[6], [18]

Different opinions of stakeholders

[8], [10]

Managerial role 


\begin{tabular}{|c|c|}
\hline Barriers & References \\
\hline $\begin{array}{l}\text { Difficulties in arriving conclusions on LCC process of a } \\
\text { building }\end{array}$ & {$[9]$} \\
\hline Deficiency of accuracy in results & [13] \\
\hline Artificiality of the process & [13] \\
\hline Lack of early-stage design applications & {$[14],[15]$} \\
\hline Lack of adjustable personal parameters & {$[10],[15]$} \\
\hline High costs of implementation & {$[1],[4],[6]$} \\
\hline Fragmented nature of the built environment & {$[9],[10]$} \\
\hline Technological barriers & [6] \\
\hline \multicolumn{2}{|c|}{$\begin{array}{l}\text { [1] (Akhlaghi, 1987), [2] (Hunter et al., 2005), [3] (Samani et al., 2018), [4] (Higham et al., } \\
\text { 2015), [5] (Heralova, 2017), [6] ( D'Incognito et al., 2015), [7] (Korpi and Ala-Risku, 2008), } \\
\text { [8] (Lindholm and Suomala, 2007), [9] (El-Haram et al., 2002), [10] (Dale, 1993), [11] (Cole } \\
\text { and Sterner, 2000), [12] (Steen, 2005), [13] (Norman, 1990), [14] (Olubodun et al., 2010), [15] } \\
\text { (Bruce-Hyrkas et al., 2018), [16] (Schmidt and Crawford, 2017), [17] (Opoku, 2013), [18] } \\
\text { (Khiyon and Mohamed, 2018) }\end{array}$} \\
\hline
\end{tabular}

\section{RESEARCH METHOD}

In the early stage of research, the background study and literature review were carried out to gain the knowledge from different sorts of resources; i.e. journal articles, conference proceedings, books, and electronic sources etc. The background study provided the basic idea of the knowledge gap and existing knowledge level related to the research problem. The literature review revealed the clear and deeper real scenario of the research problem. According to Creswell (2014), there are three research approaches as quantitative, qualitative, and mixed method. After considering the characteristic of this research, the mixed approach was identified as the best-suited approach.

Based on the findings of the literature review, to find out the barriers in practicing LCC experienced by the Sri Lankan quantity surveyors, a questionnaire survey was carried out with a quantitative approach. A closed-ended questionnaire was carried out allowing both manual and e-based responding options. Accordingly, 120 qualified quantity surveyors from different professional levels were selected as the sample through personally made requests. Ultimately, 85 dully filled questionnaires were collected with a response rate of $70.83 \%$. The research sample of 85 quantity surveyors contains various designation categories of quantity surveying professions and quantity surveyors with range of experience levels, with the aims of gathering more accurate data. Accordingly, 18 assistant quantity surveyors, 41 quantity surveyors, 14 senior quantity surveyors, and 10 chartered quantity surveyors contributed with their input to the research. Further, 20 professionals out of 85 respondence are having more than ten years' experience and 29 professionals out of 85 are having more than five years' experience. Based on the 'Mean Weighted Rating' formula (Equation 01), quantitative data were analysed.

$$
\text { Mean Weighted Rating }=\frac{\sum\left(V_{i} x F_{i}\right)}{n}
$$

Where, $\mathrm{V}_{\mathrm{i}}$-Rating given by the respondent, $\mathrm{F}_{\mathrm{i}}$ - Frequency of responses, and $\mathrm{n}$ - Total number of responses. 
After analysing the questionnaire survey data, a series of expert interviews was carried as the qualitative approach to elaborate the barriers and provide necessary mitigation actions for the same. The population for this study was considered only for charted quantity surveyors, working in the building construction industry in Sri Lanka. For this research, the target populations were limited to chartered quantity surveyors of both genders working at Colombo district. Using the convenience random sampling method, four (04) charted quantity surveyors working in consultancy services of building sector was selected. Table 2 shows the general information of the participated interviewers.

Table 2: General information of the interviewers

\begin{tabular}{clc}
\hline Interviewee & \multicolumn{1}{c}{ Position } & Experience \\
\hline R1 & Chartered Quantity Surveyor & 30 Years \\
R2 & Chartered Quantity Surveyor & 26 Years \\
R3 & Chartered Quantity Surveyor & 23 Years \\
R4 & Chartered Quantity Surveyor & 18 Years \\
\hline
\end{tabular}

\section{DATA ANALYSIS AND RESULTS}

\subsection{QUANTITATIVE DATA ANALYSIS}

The analysis of the collected data through the questionnaire survey is presented and discussed in the below section.

\subsubsection{Barriers for Practicing LCC in Building Construction Consultants}

LCC concept provides various benefits through its vivid applications in the construction industry context. However, the above literature review revealed 25 barriers for adopting LCC in building construction industry. According to the respondent's feedback, mean weight rate was calculated for each identified barrier. The analysis found lack of previous data, lack of awareness on LCC techniques by employers and practitioners, lack of common LCC calculation method, lack of industrial standards for reporting LCC outcome, lack of knowledge regarding LCC techniques, lack of formal guidelines, and lack of interest in the employers and practitioners as the common barriers for Sri Lanka building construction consultants in practicing LCC techniques. Table 3 presents the barriers of practising LCC techniques by quantity surveyors in Sri Lankan construction industry.

Table 3: Barriers in practising LCC in Sri Lankan building construction industry

\begin{tabular}{clc}
\hline Rank & \multicolumn{1}{c}{ Barriers } & Mean Weighted Rate \\
\hline 01 & Lack of awareness on the LCC tool by employers \& practitioners & 1.38 \\
02 & Lack of knowledge on LCC & 1.30 \\
04 & Lack of previous data & 1.29 \\
05 & Lack of industrial standards for reporting the LCC & 1.15 \\
06 & Lack of motivation/interest from employer \& practitioners & 1.11 \\
07 & Lack of formal guideline & 1.07 \\
08 & Technological barriers & 1.00 \\
\hline
\end{tabular}




\subsection{QUALITATIVE DATA ANALYSIS}

This section presents the qualitative data analysis in which the data were gathered through expert interviews. Based on the analysis of collected data of the questionnaire survey, expert interviews were mainly focused to obtain practical solutions for the identified barriers in quantitative analysis.

\subsubsection{Solutions for the Identified Barriers}

The following barriers have been identified as barriers for building construction consultants in practicing LCC techniques in Sri Lankan construction industry. The solutions for identified barriers are discussed below.

- Lack of awareness on the LCC tool by employers and practitioners

$\mathrm{R} 2$ expressed, "enhancing the practice of $L C C$ " is the solution for lack of awareness on the LCC tool by employers and practitioners. Further, all the interviewees mentioned that conducting new awareness programmes regarding LCC as the commonly accepted solution for the lack of awareness on LCC by employers and practitioners. In addition, R2 stated "introducing various user-friendly tools, applications and systems" also as a solution for the same barrier. Beside from that, R3 and R4 interviewees expressed the importance of including LCC techniques as a module in education courses.

- Lack of knowledge on LCC concept

According to all the respondents, the solutions for the lack of knowledge on LCC concept is, conducting courses on LCC for the employers and practitioners. In addition, R2 stated that enhancing the practice of LCC is also helpful. Moreover, R3 added that "by showing the comparison of LCC in different options and show the long-term savings of applying $L C C$ for the employers". In addition, the R4 expert said, "include the mentioned area as a module in education courses" as a solution for this barrier.

- Lack of previous data

Respondents R1, R2, R3 and R4 conveyed the necessity of maintaining a database, as a solution to this barrier. According to the perspective of R2 and R3, this database can be maintained by employers, contractors, and design consultants. However, R4 representative expressed, "in order to maintain a database in solution to this barrier, data must be collected from employers, however, employers are very reluctant to give those data". Therefore, the recognised regulatory body has to make interference to build up and maintain the database. Further, R1 and R3 stated that, CIDA, IQSSL, IESL, and SLIA are the possible regulatory bodies, who can interfere for this issue and gather cost data for building up the database.

- Lack of industrial standards for reporting the LCC

All the interviewees mentioned that establishing proper new standards by relevant organisations or institutions as the solution for this barrier. R4 stated, "recognised regulatory body has to take the responsibility to do that". Further, R1 suggested CIDA as a regulatory body and R3 suggested SLS as probable regulatory bodies. 
- Lack of formal guidelines

All the participants stated establishing a proper, user-friendly guideline as the solution for this barrier. R3 suggested that SLS or other relevant organisations should introduce new guidelines.

- Lack of interest regarding the LCC by the employers and practitioners

R1 and R2 mentioned, introducing user-friendly tools, applications, systems, and guidelines as a solution for the mentioned barrier. In addition, R3 and R4 interviewees emphasised, the need for improving the awareness on LCC tool and benefits of applying LCC techniques. Further, R3 expressed, "implementing LCC for the project can be a high initial cost", therefore, commonly employers are reluctant to practice these techniques. Further, the participants suggested that "if it is possible to find out to capitalise long-term maintenance cost and as an investment today", it will lead to mitigate the barrier.

- Lack of common LCC computing method

R1 and R2 interviewees stated introducing user-friendly new common calculations method as a solution for this barrier. R3 expressed "new computing method can be prepared based on standard designs and LCC based designs". R4 mentioned the need for improving the education courses pertaining to this area as a module will lead to mitigate this barrier in the long run.

- Technological and software barriers

According to all interviewees, the solution for technological and software barrier is to introduce new suitable technologies and software tools for the industry. However, R1 stated "these software's are very expensive therefore it is necessary to improve the funds to adopt these technologies and software tools". In addition, R3 and R4 expressed, local practitioners and employers, are backward in adopting new technology due to their attitude and culture. Therefore, changing the practitioners and employers' attitude and the culture of local context will help to mitigate mentioned barrier.

- Lack of regulations

All respondents suggested to establish and implement the prevailing rules and regulations accordingly as a solution for the mentioned barrier. Further, R3 stated that "by changing practitioners and employer's attitude" above-mentioned barrier can be mitigated.

\section{CONCLUSIONS}

In the international context practice of LCC techniques were limited due to various barriers. Comparatively, the Sri Lankan quantity surveyors are very reluctant to practice LCC techniques due to different barriers. Lack of awareness of the LCC tool by employers and practitioners, lack of knowledge on LCC, lack of previous data, lack of interest from the employers and practitioners, lack of common LCC calculation method, lack of formal guidelines lack of industrial standard for reporting LCC are the most frequent barriers hindering practice of LCC techniques in local building construction sector. In addition, the lack of industry regulations, various technological and software tools restriction also discourage the implementation of LCC.

Moreover, the intervention of industry practitioners and government will lead to enhance the practice of LCC, mitigating the above barriers. However, most of the solutions for the 
above barriers are interconnected. Therefore, recognised professional bodies within local construction industry like CIDA, IQSSL, IESL and, SLIA can promote the practice of LCC concept in Sri Lanka building construction industry by initiating the new awareness programme focusing on the benefits and applications of LCC concept, improve the training of LCC, introducing user friendly systems, and applications of LCC and including LCC as a module of education courses. In addition, government involvement through regulations and control on the prevailing or new LCC regulations, standards and guidelines will provide the solutions for the above barriers. Further, lack of previous and live data is one of the major barriers in local and international context in practicing LCC techniques. However, a proper data base is maintained by RICS in the UK. Therefore, it is necessary to build up and maintain the proper database in the Sri Lanka to avoid this particular barrier. Adopting the UK system, the regulatory bodies in Sri Lanka must take responsibility to develop and maintain a proper data base. As the responsible regularity bodies CIDA, IQSSL, IESL, and SLIA could take up the responsibility to gather and develop cost data base.

The above solution can avoid or mitigate the above barriers while contributing to the better practice of LCC techniques by quantity surveyors in Sri Lanka.

\section{REFERENCES}

Akhlaghi, F., 1987. Life cycle costing - a tool for decision making. Facilities, 5(8), pp. 4-10.

Ashworth, A., 1993. How life cycle costing could have improved existing costing. In: Life cycle costing for construction. Taylor \& Francis, pp. 119-133.

Ashworth, A., 1996. Estimating the life expectancies of building components in life-cycle costing calculations. Structural Survey, 14(2), pp. 4-8.

Ashworth, A., Hogg, K. and Higgs, C., 2013. Willis's practice and procedure for the quantity surveyor. $13^{\text {th }}$ ed. Oxford: John Wiley \& Sons, Ltd.

Ashworth, A. and Perera, S., 2015. Cost studies of buildings. $6^{\text {th }}$ ed. New York: Routledge.

Bruce-Hyrkas, T., Pasanen, P. and Castro, R., 2018. Overview of whole building life-cycle assessment for green building certification and eco design through industry surveys and interviews. Copenhagen, Denmark, Elsevier B.V.

Bull, J., 1993. Life cycle costing for construction. London: Taylor \& Francis.

Cole, R. and Sterner, E., 2000. Reconciling theory and practice of life-cycle costing. Building Research \& Information, 28(5), pp. 368-375.

Cresswell, J.W., 2014. Research design. $4^{\text {th }}$ ed. London: Sage.

Dale, S., 1993. Introduction to life cycle costing. In: J. W. Bull, ed. Life Cycle Costing for Construction. London: Taylor \& Francis, pp. 1-22.

Davies, H., 2004. Whole life costing in practice. Stoke-on-Trent: KITB Constructing Excellence.

D'Incognito, M., Costantino, N. and Migliaccio, G.C., 2015. Actors and barriers to the adoption of LCC and LCA techniques in the built. Built Environment Project and Asset Management, 5(2), pp. 202-216.

El-Haram, M. and Horner, M., 2003. Applications of principles of ILS to the development. Construction Management and Economics, 21, pp. 283-296.

El-Haram, M.A., Marenjak, S. and Horner, M.W., 2002. Development of a generic framework for collecting whole life cost data for the building industry. Quality in Maintenance Engineering, 8(2), pp. 144-151.

Ellram, L., 1995. Total cost of ownership: An analysis approach for purchasing. International Journal of Physical Distribution \& Logistics Management, 25(8), pp. 4-23.

Gillin, E., 1973. Discounted cash flow and its uses. Building Economist, 12(2), pp. 65-70.

Heralova, R.S., 2017. Life cycle costing as an important contribution to feasibility study in construction projects. Primosten, Croatia: Elsevier. 
Higham, A., Fortune, C. and James, H., 2015. Life cycle costing: Evaluating its use in UK practice. Structural Survey, 33(1), pp. 73-87.

Hunter, K., Hari, S. and Kelly, J., 2005. A whole life costing input tool for surveyors in UK local government. Structural Survey, 23(05), pp. 346-358.

Kelly, J. and Hunter, J., 2009. Life cycle costing of sustainable design. London, RICS.

Kirk, S.J. and Dell'Isola, A.J., 2003. Life cycle costing for facilities. New York: Reed Construction Data.

Kishk, M., Al-Hajj, A., Pollock, R., Aouad, G., Bakis, N. and Sun, M., 2003. Whole life costing in construction: A state of the art review. The RICS Foundation Research Paper Series, 4(18), pp. 1-39.

Knauer, T. and Moslang, K., 2005. Environmental costs and benefits in life cycle costing. Management of Environmental Quality, 16(2), pp. 107-108.

Korpi, E. and Ala-Risku, T., 2008. Life cycle costing: a review of published case studies. Managerial Auditing Journal, 23(3), pp. 240-261.

Lindholm, A. and Suomala, P., 2007. Learning by costing sharpening cost image through life cycle costing?. International Journal of Productivity and Performance Management, 56(8), pp. 651-672.

Norman, G., 1990. Life cycle costing. Property Management, 8(4), pp. 344-356.

Olubodun, F., Kangwa, J., Oladapo, A. and Thompson, J., 2010. An appraisal of the level of application of life cycle costing within the construction industry in the UK. Structural Survey, 28(4), pp. 254-265.

Opoku, A., 2013. The application of whole life costing in the UK construction industry: Benefits and barriers. International Journal of Architecture, Engineering and Construction, 2(1), pp. 35-42.

Samani, P., Gregory, J., Leal, V., Mendes, A. and Correia, N., 2018. Life cycle cost analysis of prefabricated composite and masonry buildings: Comparative study. Journal of Architectural Engineering, 24(1), pp. 431-1076.

Schmidt, M. and Crawford, R.H., 2017. Developing an integrated framework for assessing the life cycle greenhouse gas emissions and life cycle cost of buildings. Primosten, Croatia: Elsevier Ltd.

Steen, B., 2005. Environmental costs and benefits in life cycle costing. Management of Environmental Quality: An International Journal, 16(02), pp. 107-118. 